\title{
Multilevel Cooperative Search for the Circuit/Hypergraph Partitioning Problem
}

\author{
Min Ouyang, Michel Toulouse, Krishnaiyan Thulasiraman, Fellow, IEEE, Fred Glover, and Jitender S. Deogun
}

\begin{abstract}
The objectives in this paper are twofold: design an approach for the netlist partitioning problem using the cooperative multilevel search paradigm introduced by Toulouse $e t$ al. and study the effectiveness of this paradigm for solving combinatorial optimization problems, in particular, those arising in the very large scale integration (VLSI) computer-aided design (CAD) area. The authors present a cooperative multilevel search algorithm CoMHP and describe a parallel implementation on the SGI O2000 system. Experiments on ISPD98 benchmark suite of circuits show, for four-way and eight-way partitioning, a reduction of $3 \%$ to $15 \%$ in the size of hyperedge cuts compared to those obtained by hMETIS. Bisections of hypergraphs based on the algorithm also outperform hMETIS, although more modestly. The authors present experimental results to demonstrate that the cooperation scheme plays a key role in the performance of CoMHP. In fact, the improvement in the quality of the solutions produced by CoMHP is to a large extent independent of the partitioners used in the implementation of CoMHP. The experimental results also demonstrate the effectiveness of the cooperative multilevel search paradigm for solving the netlist partitioning problem and show that the cooperative multilevel search strategy can be used as a paradigm for designing effective solution techniques for combinatorial optimization problems such as those arising in the VLSI CAD area.
\end{abstract}

Index Terms-Combinatorial optimization, cooperative search, graph partitioning, multilevel algorithms, VLSI physical design.

\section{INTRODUCTION}

$\mathbf{N}$ ETLIST partitioning is an important and well-studied research topic in the very large scale integration (VLSI) computer-aided design (CAD) area. Several classes of heuristics have been proposed to address this problem [2]. Recently, multilevel algorithms have been applied to the netlist partitioning problem [11]. This approach has since become the standard to partition netlists.

In the multilevel paradigm, Fiduccia-Mattheyses (FM) types of move-based heuristics execute moves in coarsened hypergraphs (hypergraphs are a common mathematical representa-

Manuscript received July 13, 2000; revised September 20, 2001. This paper was recommended by Associate Editor R. Gupta.

M. Ouyang is with Synopsys Inc., Mountain View, CA 94043 USA (e-mail: mouyang@ synopsys.com).

M. Toulouse is with the Department of Computer Science, University of Manitoba, Winnipeg, MB R3T 2N2, Canada (e-mail: toulouse@ cs.umanitoba.ca).

$\mathrm{K}$. Thulasiraman is with the School of Computer Science, University of Oklahoma, Norman, OK, 73019 USA (e-mail: thulasi@cs.ou.edu).

F. Glover is with the Hearin Center for Enterprise Science, School of Business Administration, University of Mississippi, University, MS 38677 USA (e-mail: fglover@bus.olemiss.edu).

J. Deogun is with the Computer Science and Engineering Department University of Nebraska-Lincoln, Lincoln, NE 68588-0115 USA (e-mail deogun@cse.unl.edu).

Publisher Item Identifier S 0278-0070(02)04702-4. tion of netlists) that involve static clusters (blocks) of modules from the original netlist instance. Since the clusters are static, the search space of coarsened hypergraphs is often a tiny fraction of the search space of the original optimization problem. This reduction in the search spaces enables a speedy execution of multilevel algorithms. Unfortunately, it also imposes serious limitations on the ability of multilevel algorithms to provide good quality partitionings. These limitations of the multilevel paradigm have been recently addressed in [7] and [11] using more dynamic coarsening strategies. In this paper, we present a broader strategy to address this issue.

Our approach is based on a bottom-up algorithm design technique called cooperative search. According to this approach, a set of different search algorithms is first selected. Each algorithm is implemented as an independent program that runs in time sharing with the other programs on a sequential computer or in parallel if several computing units are available. If the difference among the programs is only based on the stochastic properties of a generic algorithm or on different search parameters, then we can think of those programs as the multiple restarts of the same algorithm. However, unlike restart, programs in a cooperative search interact with each other based on a cooperation protocol that specifies how the search programs cooperate at run time. Intuitively, as framed in Huberman's paper [10], cooperation is an exchange of "hints" that may confuse some search processes, but will also help others. Overall, hint sharing improves the performance and has been used with success to design search heuristics in the context of constraint satisfaction problems [3], [8] and to parallelize some metaheuristics [13], [14], [16], [17].

The present paper introduces the cooperative multilevel hypergraph partitioning algorithm (CoMHP), an asynchronous variation for hypergraph partitioning of the cooperative algorithm in [18]. Our hypergraph partitioning method uses a new netlist coarsening strategy which is based on partitioning rather than clustering, as it is usually done by multilevel algorithms. Next, we introduce a cooperation protocol which supports a dynamic "recoarsening" strategy addressing the convergence problems of standard multilevel algorithms. Finally, we give an intuitive description of the convergence behavior for this system of cooperating search algorithms.

The rest of the paper is structured as follows. Section II introduces a few definitions and our coarsening strategy. Section III describes the cooperative algorithm and its convergence behavior. Section IV reports and discusses the results of the tests conducted on the ISPD98 benchmark suite of circuits. Finally, Section V concludes with some suggestions for future work. 


\section{BASIC DEFINITIONS AND PARTITION-BASED COARSENING}

Hypergraphs are commonly used as a formal representation of netlists. Let $H_{0}=\left(V_{0}, E_{0}\right)$ be a hypergraph representation of a given netlist instance. $V_{0}$ is a set of $n$ vertices and $E_{0}$ a set of $m$ hyperedges which represent, respectively, the modules or vertices and signal nets of the netlist. The set $E_{0}$ is a subset of the powerset $2^{V_{0}}$ of the vertices in $H_{0}$, i.e., $e \in E_{0}$ is a subset of $V_{0}$. Note that the superset of a set $S$ is defined as the collection of all subsets of $S$. Given this formalization, the problem of partitioning the modules of a netlist into $k$ subsets $P_{1}, P_{2}, \ldots, P_{k}$ can be stated as a combinatorial optimization problem where one tries to find an instance $\left\{P_{1}, P_{2}, \ldots, P_{k}\right\}$ of the mapping

$$
\mathcal{P}: V_{0} \longrightarrow 2^{V_{0}}
$$

that minimizes the cost function

$$
f(x)=\sum_{i=1}^{m} w\left(e_{i}\right)
$$

where $w\left(e_{i}\right)=1$ if $e_{i}$ is a hyperedge that spans more than one $P_{i}$, and $w\left(e_{i}\right)=0$, otherwise. The subsets $P_{i}$ are subject to the constraints:

1) $P_{i} \cap P_{j}=\emptyset(i \neq j)$;

2) $\left|V_{0}\right| / c k \leq\left|P_{i}\right| \leq c\left|V_{0}\right| / k$ for some constant $c \geq 1.0$;

3) $\bigcup_{i=1}^{k} P_{i}=V_{0}$.

Constraints 1) and 3) ensure that $\left\{P_{1}, P_{2}, \ldots, P_{k}\right\}$ is a partition and constraint 2) sets bounds on the cardinalities of $P_{i}$ in the partitions. The set of instances from Mapping (1) satisfying these constraints are identified as the solution space $X_{0}$ of this problem. The above problem is a generalization of the graph partitioning problem, an NP-complete problem [6]. Consequently, search heuristics are handy to provide good solutions in reasonable computational time.

Multilevel algorithms initiate processing by a hierarchical clustering of the vertices of $H_{0}$, yielding hypergraphs with fewer vertices, i.e., coarsened hypergraphs. Usually, recursive matching-based clustering algorithms such as edge-coarsening, hyperedge-coarsening, maximal matching, and modified-hypergraph-coarsening [4], [11] are used to coarsen hypergraphs. Our clustering strategy addresses the problem of contracting the netlist as a partitioning problem. In this paper, hierarchical clustering and coarsened hypergraph are defined in the following manner.

Definition 1: Let

$$
\mathcal{C}_{i}: V_{0} \longrightarrow 2^{V_{0}}, \quad i=1, \ldots, l
$$

be a family of $l$ mappings where $\mathcal{C}_{i}$ maps the vertices of $H_{0}$ into $\left|V_{i}\right|$ clusters $C_{i 1}, C_{i 2}, \ldots, C_{i\left|V_{i}\right|}$ such that $C_{i u} \cap C_{i v}=\emptyset$ if $u \neq v$ and $\bigcup_{j=1}^{\left|V_{i}\right|} C_{i j}=V_{0}$. The family of mappings in (3) defines a hierarchical clustering of the vertices of $H_{0}$ whenever $\left|V_{i}\right|>\left|V_{i+1}\right|$ for all $i=1,2, \ldots, l-1$.

Definition 2: A coarsened hypergraph $H_{i}=\left\{V_{i}, E_{i}\right\}$ is the set of vertices $V_{i}$ and hyperedges $E_{i}$ such that: 1) $v \in V_{i}$ is a cluster (subset) $C_{i j}$ of vertices from $V_{0}$ as defined by the mapping $\mathcal{C}_{i}$ (Note: to simplify notation, $v$ may also be denoted by the corresponding cluster $C_{i j}$.) and 2) a subset $e$ of $V_{i}$ is a hy- peredge of $H_{i}$ if and only if there exists a hyperedge $e^{\prime} \in E_{0}$ such that $e^{\prime}$ has nonempty intersection with each cluster represented by the vertices in $e$.

Basically, the above definition formally states how coarsened hypergraphs $H_{i}$ are formed; each cluster in the partition defined by the mapping $\mathcal{C}_{i}$ represents a vertex of $H_{i}$ and a hyperedge $e$ of $H_{0}$ becomes an appropriately modified hyperedge of $H_{i}$ if the vertices in $e$ are not all in the same cluster of the partitioning defined by $\mathcal{C}_{i}$. For instance, if each hyperedge of $H_{0}$ is a pair of vertices in $V_{0}$, then for $u \neq v, e=\left\{C_{i u}, C_{i v}\right\} \in E_{i}$ iff $\exists a, b \in V_{0}$ such that $a \in C_{i u}$ and $b \in C_{i v}$ and $\{a, b\}$ is a hyperedge in $E_{0}$.

In our hierarchical clustering approach, each mapping $\mathcal{C}_{i}$ is obtained by solving a hypergraph partitioning problem for $H_{0}$. The family of mappings as defined in (3) is derived from the solution of the $n / 2^{i}$-way partitioning problems for $H_{0}$, for $0<i \leq l$. The solution of the $n / 2^{i}$-way partitioning, i.e., the $n / 2^{i}$ subsets, become the set of vertices $V_{i}=\left\{C_{i 1}, C_{i 2}, \ldots, C_{i\left(n / 2^{i}\right)}\right\}$. Next the coarsened hypergraphs $H_{i}$ are generated from the mappings $\mathcal{C}_{i}$ as described in Definition 2 . We identify this hierarchical coarsening strategy as partition-based coarsening. This algorithm can be trivially parallelized by running the partitioning processes and the hypergraph generations on different processors. The parallel time requirement is dominated by the processor that computes the partitioning for $k \approx n / 2$.

\section{CoMHP: DESCRIPTION AND CONVERGENCE BEHAVIOR}

In this section, we present in detail, the different components of our multilevel cooperative search algorithm, CoMHP, and a model to study its convergence behavior. There are $l+1$ processes in CoMHP, the same as the number of hypergraphs. Each process $p_{i}$ takes as input the hypergraph $H_{i}$. The processes run in parallel, applying the same composition of FM-like hypergraph partitioning heuristics to partition their hypergraph. According to Definition 1 , moving a vertex $v$ in a coarsened hypergraph $H_{i}$ is equivalent to moving a cluster of vertices in $H_{0}$, the cluster corresponding to vertex $v \in H_{i}$. Consequently, while a process $p_{i}$ searches for good partitionings in the coarsened hypergraph $H_{i}$, it actually explores the solution space $X_{0}$. Though all the processes use the same local search methods, the searches in $X_{0}$ do not overlap completely. This is because, as in standard multilevel algorithms, hierarchical clustering in CoMHP creates different neighborhoods to solutions in $X_{0}$.

The computational cost of executing a move in neighborhoods based on coarsenings is independent of the size of the clusters in $H_{0}$. It only depends on the number of neighbors of the current solution; this number goes on decreasing as the hypergraphs are more highly coarsened. This is a clear advantage of these neighborhoods. On the other hand, if the coarsening cannot be undone, the logical moves in $H_{0}$ only involve the clusters of vertices in $H_{0}$ associated by the Mapping (3). All the possible combinations of such clusters in $k$ subsets can only generate a small fraction of the solutions in $X_{0}$. This is not a problem by itself since it is usual for local searches to explore only a fraction of the solution space. Rather, the problem is when the small search spaces of coarsened hypergraphs have no good 
solutions, their exploration is then hopeless, even using the best search methods.

This limitation of the coarsening approach has in fact been partially identified in [7], [11] by observing that an initial partitioning can be refined in different ways depending upon how the coarsening is executed [11]. Those papers propose a multiphase refinement as a mechanism to refocus the search spaces. Such multiphase refinement is based on a recursive call of the multilevel algorithm from the same coarsened hypergraph in the multilevel structure. A randomized coarsening is used during the multiphase refinement. In [11], this coarsening is initiated from the best partitioning obtained from the refinement or from the previous iteration of the multiphase refinement. The multiphase refinement iterates until the best solution cannot be improved further. The initial refinement phase of the multilevel procedure is then resumed.

Our multilevel cooperative algorithm is similar in spirit to multiphase refinement. It also produces many "recoarsenings" of the hypergraphs which allow the search processes to explore new regions in the solution space $X_{0}$.

\section{A. Refinement Phase}

The initial coarsening may fail to provide access to good regions of the solution space. We now describe how the partitionings discovered by the search heuristics during the refinement phase can be used to develop a dynamic coarsening strategy. Our dynamic coarsening strategy is supported by the cooperation protocol of CoMHP. This protocol specifies the processes' neighborhood structure, the kind of interactions (hint exchanges) allowed among search processes, and what to do with the hints exchanged.

The neighborhood structure in the current implementation is an array of processes: process $p_{i}$ can only interact with processes $p_{i-1}$ and $p_{i+1}$. This neighborhood structure mirrors the hierarchical clustering of $H_{0}$, where $H_{i}$ has for neighbors hypergraphs $H_{i-1}$ and $H_{i+1}$ in the sequence of increasingly coarsened hypergraphs. The boundary conditions in the array are handled in the following manner: $p_{0}$ only interacts with $p_{1}, p_{l}$ only interacts with $p_{l-1}$.

Each interaction involves exchange of "hints" or information based on "elite partitionings" and taking appropriate actions. Elite partitionings are those that we expect to give us hints as to the nature of optimum partitionings. For instance, a partitioning, which has the smallest hyperedge cut among all those partitionings generated by the search method used, may be considered elite at that stage. Let $X_{i}$ be the search space of $H_{i}$, whereas $X_{i}^{\prime}$ is the set of elite partitionings of $H_{i}$. Different approaches have been tested to create each elite set $X_{i}^{\prime}$ of hypergraph $H_{i}$. The approach presented in Fig. 4 initializes the elite set to a good partitioning generated by a search method. A newly generated partitioning is added to $X_{i}^{\prime}$ if its hyperedge-cut value is not larger than $10 \%$ of the smallest of the hyperedge cuts of the partitionings already in the set $X_{i}^{\prime}$. We also limit the size of $X_{i}^{\prime}$. If the addition of a partitioning increases this limit, we remove from $X_{i}^{\prime}$ the partitioning which has the highest hyperedge-cut value among all those already in the set $X_{i}^{\prime}$.

The operators, to be described soon, specify how to use the elite partitionings at the level of each process. The local parti-

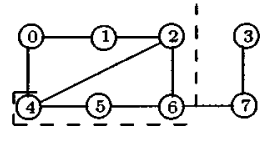

$\mathbf{H}_{0}$

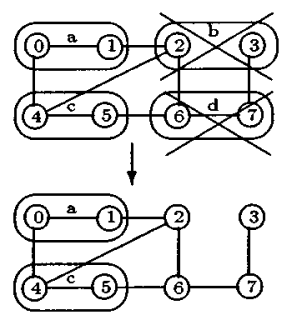

(b)

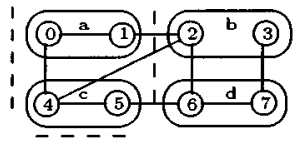

$\mathrm{H}_{1}$

(a)

(c)

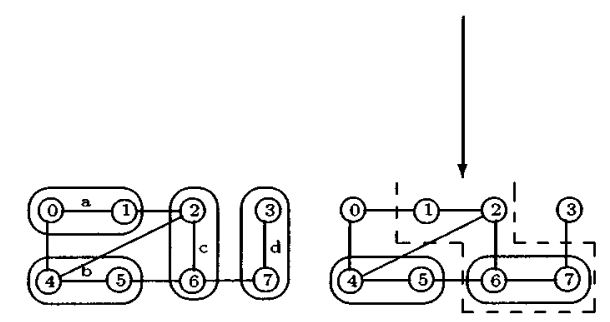

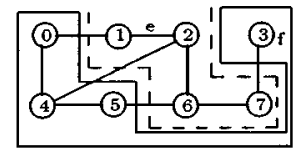

$\mathrm{H}_{2}$ (d)

Fig. 1. Interaction operators: (a) coarsened hypergraphs (dashed lines indicate partitionings), (b) local partitioning operator, (c) local clustering operator, and (d) interpolation operator.

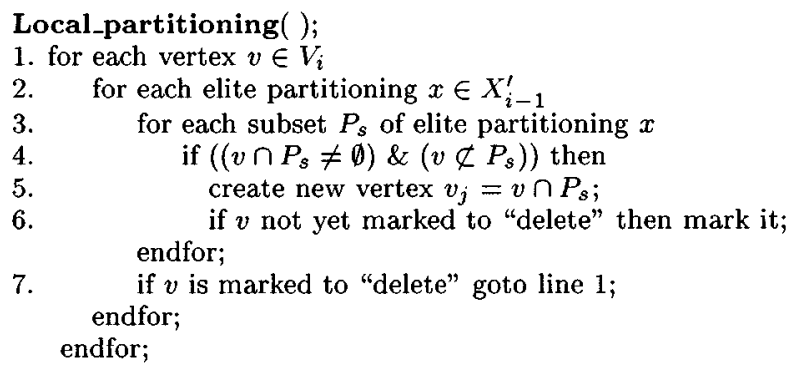

Fig. 2. Pseudocode of the local partitioning operator.

tioning operator and the local clustering operator redefine the coarsening of hypergraphs. On the other hand, the interpolation operator reinitiates the search of some of the move based heuristics used by each process. More specifically, in the interpolation operator, one elite partitioning from $H_{i+1}$ is selected as the initial solution of a move based heuristic in hypergraph $H_{i}$. We now describe, in detail, these three operators. Fig. 1 is used for illustration of the workings of these operators. Fig. 1(a) gives $H_{0}, H_{1}$, and $H_{2}$ with one elite partitioning for each indicated by dashed lines.

1) Local Partitioning Operator: Local partitioning changes the coarsening of $H_{i}$ by splitting some of its vertices. Vertices (clusters) in $H_{i}$ are split based on the information provided by the set of elite partitionings $X_{i-1}^{\prime}$ from hypergraph $H_{i-1}$. The local partitioning operator finds clusters $v \in V_{i}$ (line 1 in Fig. 2) such that $v$ has at least two vertices $a, b \in V_{0}$ that are into two different subsets (line 4) of one of the elite partitionings of $X_{i-1}^{\prime}$. When this happens, the vertices of $H_{0}$ in the intersection of the sets $v \cap P_{s}$ form a new vertex $v_{j}$ of $H_{i}$ (line 5). Once the local partitioning operator has been completed, a special routine is called which generates a new coarsened hypergraph $H_{i}$ reflecting the changes in the mapping $\mathcal{C}_{i}$, provoked by the execution of the local partitioning operator. Note that in the pseudocode of Fig. 2, after the first elite partitioning for which we find at least one nonempty intersection with a subset in this partitioning, we end the "for loop" (beginning at line 1) for this vertex. In other words, we split a vertex with respect to only one elite partitioning, if possible. 
We shall first illustrate the local partitioning operator with a hypothetical example. Then the illustration will be given using Fig. 1.

Consider the hypergraph $H_{i}$. Pick any vertex $v$ of $H_{i}$ (line 1 in Fig. 2). Note that $v \in V_{i}$ is a cluster of vertices of $H_{0}$. Let $v=\{2,7,8,9\}$. Here 2, 7, 8, and 9 are all vertices of $H_{0}$. Consider an elite partitioning $x$ of $H_{i-1}$ (line 2 in Fig. 2). Here, we assume that the optimization problem is to search for optimum three-way partitionings. Let the three subsets in the partitioning $x$ be $P_{1}=\{1,2,3,8,12\}, P_{2}=\{9,11,13,14\}$, and $P_{3}=\{4,5,6,7,10\}$. Now we get (line 4 in Fig. 2)

$$
v \cap P_{1}=\{2,8\}, \quad v \cap P_{2}=\{9\}, \quad \text { and } \quad v \cap P_{3}=\{7\} .
$$

We then create the following new vertices (line 5 in Fig. 2) as a result of splitting vertex $v$ of $H_{i}$ :

$$
\{2,8\}, \quad\{9\}, \quad \text { and } \quad\{7\} \text {. }
$$

Once we identify a partitioning $x$ (as above) and split vertex $v$, then $v$ is marked to be deleted (line 6) and the operator returns to line 1 to pick a new vertex and check if it could be split as above. In case the elite partitioning $x$ does not help to split the vertex $v$, we pick another partitioning from the list of elite partitionings $X_{i-1}^{\prime}$. If no elite partitioning leads to a splitting of $v$, then this vertex $v$ in $H_{i}$ is left unchanged as it was before the execution of the local partitioning operator. The algorithm for the local partitioning operator terminates once all the vertices of $H_{i}$ have been considered for possible splittings.

We now return to Fig. 1 for another illustration of the local partitioning operator with a concrete example. Consider the hypergraph $H_{1}$ of Fig. 1(a). (Vertices of coarsened hypergraphs as well as the subsets of partitionings are sets of vertices from the hypergraph $H_{0}$.) This hypergraph has four vertices $a, b, c, d \in$ $V_{1}$, where $a$ is a cluster $\{0,1\}$ of vertices of $H_{0}, b$ is a cluster $\{2,3\}$ of vertices of $H_{0}$, and so on. The elite bisection of $H_{0}$ is $P_{1}=\{0,1,3,7\}$ and $P_{2}=\{2,4,5,6\}$. This elite bisection indicates that potentially good solutions exist in the region of the solution space in which vertices 2 and 3 are in the different subsets of each bisection. But in $H_{1}$, vertices 2 and 3 form a cluster; swaps on $H_{1}$ always move vertices 2 and 3 of $H_{0}$ together so that they are in the same subset of the bisection of $H_{1}$ because they form a single vertex in $H_{1}$. The region of the solution space corresponding to the elite bisection of $H_{0}$ is not reachable in the search space defined by the coarsened hypergraph $H_{1}$. Although the search space of $H_{1}$ is smaller than $X_{0}$ and therefore faster to explore, this advantage is lost because search space $X_{1}$ does not overlap with good solutions of the basic optimization problem. This problem is detected by the local partitioning operator because the intersection of the sets $b$ and $P_{1}$ is not empty and $b$ is not strictly included in $P_{1}$. Similarly, the intersection of the sets $b$ and $P_{2}$ is nonempty and $b$ is not strictly included in $P_{2}$. Also, the intersections of vertex $d=\{6,7\}$ with $P_{1}$ and $P_{2}$ are nonempty. In other words

$$
\begin{aligned}
& b \cap P_{1}=\{3\} \quad \text { and } \quad b \cap P_{2}=\{2\} \\
& d \cap P_{1}=\{7\} \quad \text { and } \quad d \cap P_{2}=\{6\} \text {. }
\end{aligned}
$$

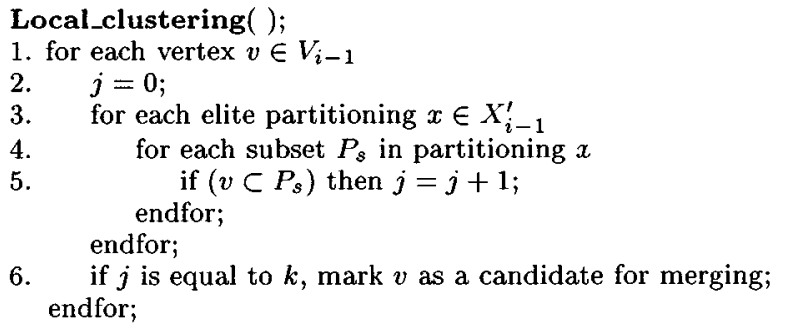

Fig. 3. Pseudocode of the local clustering operator.

So, the vertex $b$ is split into the two vertices $\{3\}$ and $\{2\}$, and the vertex $d$ is split into the two vertices $\{7\}$ and $\{6\}$. Note that the vertices $a$ and $c$ are not split because they are strictly included in $P_{1}$ and $P_{2}$, respectively. The resulting coarsening of $H_{1}$ is shown in Fig. 1(b). Note that this new coarsening of $H_{1}$ enables the search space $X_{1}$ to overlap with the regions of $X_{0}$ identified by the elite bisection.

2) Local Clustering Operator: Local clustering changes the coarsening of $H_{i}$ by merging some of the vertices (clusters) of $H_{i-1}$ into new vertices for $H_{i}$. A vertex from $H_{i-1}$ becomes a "candidate for merging" if it is strictly included in one of the subsets of each elite partitioning from $X_{i-1}^{\prime}$ (line 5 in Fig. 3). The identification of which vertex can be a candidate for merging is needed because between the time a partitioning enters the set of elite partitionings and the time local clustering is run, many vertices of $H_{i-1}$ may have been split or clustered such that some of them may overlap more than one subset of an elite partitioning. According to the definition of (2), a vertex is strictly included in exactly one subset of a partitioning. Therefore, if $j=k$ in line 6 , vertex $v$ is strictly included in one subset of each elite partitioning. Once the candidates for merging have been identified, a pair of vertices can be merged if both vertices satisfy the two following conditions: 1 ) both vertices lie on the same hyperedge and 2) both vertices are together in the same subset in all elite partitionings. Note that two vertices $v_{1}$ and $v_{2}$ of $H_{i-1}$ are on the same hyperedge $e$ of $H_{0}$ if they are both strictly included in $e$. For instance, in the case each hyperedge is a pair of vertices, then $v_{1}$ and $v_{2}$ are on the hyperedge $e$ if $e=\left\{v_{1}, v_{2}\right\}$. Once a vertex is identified as candidate for merging, the vertex is labeled with the subset of each elite partitioning where it has been found. Vertices with the same label satisfy condition 2 above.

We shall next illustrate the local clustering operator with a hypothetical example. Consider vertices $v_{1}$ and $v_{2}$ of $H_{i-1}$ (line 1 in Fig. 3). Let $v_{1}=\{6,8\}$ and $v_{2}=\{12,13\}$, and let $x_{1}, x_{2}$ and $x_{3}$ be the three elite partitionings of $H_{i-1}$. That is, they form the set $X_{i-1}^{\prime}$. Assuming three-way partitionings, let the three subsets in $x_{1}, x_{2}$ and $x_{3}$ be

$$
\begin{aligned}
& x_{1}=\{2,7,9,11\},\{1,3,5,10\},\{4,6,8,12,13,14\} \\
& x_{2}=\{1,3,4,5,10\},\{2,7,9,11,14\},\{6,8,12,13\} \\
& x_{3}=\{6,8,12,13,\},\{2,7,9,11,14\},\{1,3,4,5,10\} .
\end{aligned}
$$

We can see that the vertex $v_{1}=\{6,8\}$ is strictly included in one of the subsets in each of the above elite partitionings (lines 3, 4, 5 in Fig. 3) and so it is eligible for merging. Similarly, the 
vertex $v_{2}=\{12,13\}$ is also eligible for merging. If $e=\{3,6$, $8,9,10,12,13\}$ is a hyperedge, then both $v_{1}$ and $v_{2}$ lie on this hyperedge, and so we can merge them to form a vertex $\{6,8$, $12,13\}$ for the hypergraph $H_{i}$.

Now we return to Fig. 1 for another illustration of the local clustering operator with a concrete example. According to the elite bisection of $H_{0}$ [see Fig. 1(a)], vertices 0 and 1 are in the same subset of this elite bisection. These vertices also lie on the same hyperedge. So, they are merged to form vertex $a$ of $H_{1}$ [see Fig. 1(c)]. For similar reasons, we merge vertices 4 and 5 to form vertex $b$ of $H_{1}$, merge vertices 2 and 6 to form vertex $c$ of $H_{1}$, and merge vertices 3 and 7 to form vertex $d$ of $H_{1}$. These vertices $a, b, c$, and $d$ now define the new coarsened hypergraph $H_{1}$.

Local clustering tends to reduce the number of vertices in a coarsened hypergraph and therefore to reduce the size of the search space. This balances the effect of the local partitioning operator which tends to increase the number of vertices. It also makes the search space retreat from overlapping with uninteresting regions of the solution space. Together, local partitioning and local clustering operators allow the search spaces to move in the solution space, which is the desired effect of recoarsening.

3) Interpolation Operator: In the interpolation operator, one partitioning from the set of elite partitionings of $H_{i+1}$ is selected to be the initial solution of a move-based heuristic in hypergraph $H_{i}$. Our interpolation operator would be identical to the interpolation operator of multilevel algorithms if it was not for the way we compute the coarsened hypergraphs. Recursive coarsening used by most multilevel algorithms is such that vertices $C_{i j} \in H_{i}$ are formed by the clustering of two vertices from $H_{i-1}$. For example, when vertices $u, v \in H_{0}$ are mapped together to an aggregate $C_{(i-1) j} \in H_{i-1}$, those two vertices are necessarily mapped to $C_{i j}$, the superset of $C_{(i-1) j}$ in the coarsened hypergraph $H_{i}$. Hypergraphs generated with recursive coarsening are said to be related level by level. This is not necessarily the case with our coarsening strategy. The vertices of $H_{0}$ that are mapped to a cluster $C_{i j} \in H_{i}$ may be spread over several clusters in each hypergraph $H_{j}, j>i$.

Returning to Fig. 1, assume the elite partitioning of hypergraph $\mathrm{H}_{2}$ is selected as initial solution for a move-based heuristic of process $p_{1}$ associated with hypergraph $H_{1}$. The elite partitioning cannot be used because hypergraphs $H_{1}$ and $H_{2}$ are not related level by level. For example, vertex $a$ of $H_{1}$ spreads over vertices $e$ and $f$ of hypergraph $\mathrm{H}_{2}$ (same thing for $b$ ). The elite partitioning of $\mathrm{H}_{2}$ cannot be a partitioning for the hypergraph $H_{1}$. In order to use the partitioning from $\mathrm{H}_{2}$, we change the coarsening of $H_{1}$. A split of vertices in $H_{1}$ that spreads over more than one vertex in $H_{2}$ is performed using a similar procedure as for the local partitioning operator. That is, we consider splitting the vertices $a, b, c$, and $d$ of $H_{1}$ [see Fig. 1(a)] using the bisection of $H_{2}$ shown in Fig. 1(a). As shown in Fig. 1(d), after the split, it becomes possible to use the elite partitioning from $\mathrm{H}_{2}$ as an initial solution to one of the move-based heuristics in our search method.

4) The Search Heuristic of CoMHP's Processes: We are now ready to describe the search heuristic run by the $l+1$ search processes of CoMHP. This search heuristic combines, in a single iterative loop, several hypergraph partitioning heuris-

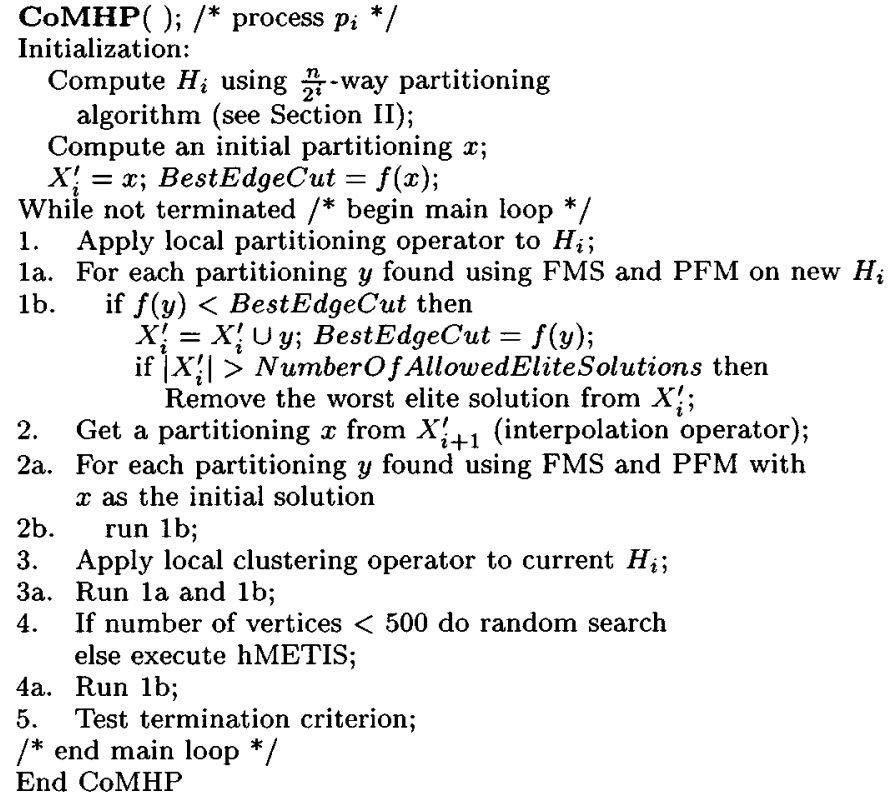

Fig. 4. CoMHP for process $p_{i}$.

tics and the three operators that handle hints from neighbor processes. Each iteration of the loop executes the following sequence of operations: local partitioning, local searches, interpolation, local searches, local clustering, local searches, and global searches. Fig. 4 contains an abbreviated pseudocode for this loop for process $p_{i}$.

The initialization phase computes a coarsened hypergraph and an initial partitioning. The initial partitioning is required to provide a first elite solution to the set $X_{i}^{\prime}$ and an initial solution to the iterative local search methods used in the main loop (unlike constructive methods, iterative methods have to be provided with an initial solution that they then improve).

The execution sequence of the interaction operators (local partitioning, interpolation, local clustering) has been chosen arbitrarily. However, once the local partitioning operator is performed at level $i$, hypergraph $H_{i}$ is modified. We then run iterative local search methods (line 1a) on this new hypergraph $H_{i}$ to calculate new hyperedge cuts as well as to discover potential elite solutions (line 1b). As local search methods we have used the Sanchis partitioning algorithm (FMS) [15], and the multiway partitioning by free moves (PFM) proposed by Dasdan and Aykanat [5]. Next, on line 2, the interpolation operator is applied to get an elite solution $x$ from $X_{i+1}^{\prime}$, so that $x$ can be used as an initial solution for the search methods FMS and PFM. Finally, the local clustering operator is applied, which transforms $H_{i}$ once again, allowing us to repeat the execution of FMS and PFM on a different hypergraph $H_{i}$.

The search of line 4 in Fig. 4 serves two different purposes. For highly coarsened hypergraphs, less than 500 vertices for example, several random searches could be executed. As these hypergraphs are very small, the main loop runs very fast and the process may run out of elite partitionings from neighboring processes. By executing many random searches, we slow down the execution of the main loop while having chances to discover good partitionings. The second purpose is to execute a search of $H_{i}$ which does not depend on any initial partitioning. Both 
the hMETIS software and random searches have this characteristic (our random search routine consists of an initial partitioning generated randomly, followed by the execution of an iterative search to refine the random partitioning).

All the processes run the same combination of heuristics and interaction operators, except for the special conditions that hold for the boundaries processes $p_{0}$ and $p_{l}$ and for the difference between highly coarsened hypergraphs and the other hypergraphs.

CoMHP is an asynchronous algorithm in the sense that each process executes the iterative loop of the search method without synchronization with other processes. Therefore, processes associated with highly coarsened hypergraphs run more iterations of the search method compared, for example, to the process associated with $H_{0}$. The time requirement for the cooperation protocol is the same for all processes. Furthermore, this time requirement is insignificant compared to the time required by the search heuristics.

\section{B. Convergence Behavior of CoMHP}

Because of the local interactions among the search processes, the convergence behavior of cooperative algorithms is sometimes modeled according to the theory of complex systems. For example, Huberman [10] uses a probabilistic model to show that the performance of cooperating processes is log-normally distributed for successful cooperative algorithms, in contrast with the normal distribution of independent searches (or restarts). In this model, the effect of cooperation on the distribution is a smaller number of average quality searches but an increase of the length of the tails on both sides of the distribution. The long tail of the positive side of the distribution produces the overall performance improvement. But to achieve such performance improvement, the cooperative procedure must satisfy the following requirements.

1) A large set of heuristically guided searches.

2) The searches apply successfully different search strategies, leading to nonredundant explorations of the solution space.

3) Processes exchange some useful information (hints) that allows some of them to cut the number of steps required to reach an optimal or acceptable solution.

4) Hints are statistically independent.

Requirements 1 and 2 are necessary to provide statistical independence among the hints. In practice, however, these requirements often conflict with one another. Either the number of guided searches is too small or the explorations of the different searches overlap in the solution space. When this happens, cooperative programs do not provide consistent quality of solutions: they converge well on some instances, yet very poorly on other instances of the same optimization problem.

Besides addressing the limitations induced by static coarsened hypergraphs in standard multilevel algorithms, a second motivation for mixing the cooperative paradigm with the multilevel paradigm has been to address the issue of conflicting requirements facing many implementations of cooperative algorithms. In the context of cooperative search, the stability of coarsened hypergraphs helps to reduce the negative influence that suboptimal hints can have on the convergence behavior of the system. Hints have first to change the static neighborhood structures defined by coarsened hypergraphs before directing the search in new regions of the solution space. This is unlike any other cooperative algorithms. We believe that this is the reason why the best solutions found by CoMHP are very close to those of cooperative procedures that meet the requirements above. However, the performance of cooperating processes in CoMHP does not settle in a log-normal distribution because there are usually too few processes and hints are often strongly correlated. Rather, the cooperation protocol in CoMHP is such that it tends to minimize the differences between the hyperedge cuts of the elite partitionings. The evolution of CoMHP settles in a minimum energy state (such as Hopfield networks [9]). The energy function is given by the sum of the differences between the averages for the hyperedge cuts $f(x)$ of the elite partitionings $x$ of the different processes

$$
E(\mathcal{X})=\sum_{i=0}^{l-1}\left\{\frac{\sum_{x \in X_{i}^{\prime}} f(x)}{\left|X_{i}^{\prime}\right|}-\frac{\sum_{x \in X_{i+1}^{\prime}} f(x)}{\left|X_{i+1}^{\prime}\right|}\right\} .
$$

The initial state of the system is given by the elite partitionings computed after the coarsening phase. If the coarsening phase is successful, the elite partitionings from neighboring processes will have different hyperedge cuts. The differentials among the best hyperedge cuts of neighbor processes create opportunities to change, by percolation, the coarsening of the neighbor hypergraphs. New coarsenings provide new elite partitionings which in turn affect the coarsening of neighboring hypergraphs. This percolation process stops to have an impact on the exploration of the solution space when all the elite partitionings have about the same hyperedge cuts, which corresponds to a minimum energy level of the system. Once the system has reached such a minimum energy state, the quality of the best partitioning does not improve much. In terms of the best partitioning, when the system [as modeled in (4)] is stable, that is, it has reached a fixed point, the computation can then be ended.

\section{EXPERIMENTAL RESULTS}

We have evaluated the performance of our CoMHP algorithm on the ISPD98 benchmark suite of netlists [1], comparing the performance of CoMHP with version 1.5.3 of the hMETIS partitioning package. We have implemented a parallel version of our hypergraph partitioning algorithm and have run it on the SGI computer at the Research Computing Facility (RCF) of the University of Nebraska-Lincoln. hMETIS has also been run on this same environment. RCF possesses a shared memory SGI O2000 system with 16 250-Mhz R10k CPUs, 4-GB main memory, and runs on the IRIX 6.5 Operating System. For each problem instance, we have executed ten runs of hMETIS with recursive bisection and ten runs with hMETIS-Kway (the direct approach) [12]. Our algorithm has been run for ten iterations of process $p_{0}$. Since hypergraph $H_{0}$ is the largest one in the sequence of hypergraphs, process $p_{0}$ takes more time than any other process to complete one iteration of the refinement phase.

Tables I and II present the two-, four-, and eight-way hyperedge cuts for, respectively, the unit cell area and the nonunit 
TABLE I

Min-Cut Two-, Four, Eight-Way Partitioning Results With UP to A 10\% Deviation From Exact Partitioning. Cells Are Assigned UNIT AREA (COLUMNS "hM" AND "Co" STAND, RESPECTIVELY, FOR hMETIS AND CoHMP)

\begin{tabular}{l||c|c|c|c|c|c}
\hline \multicolumn{1}{c||}{ Circuit } & \multicolumn{2}{c|}{ 2-way } & \multicolumn{2}{c|}{ 4-way } & \multicolumn{2}{c}{ 8-way } \\
\cline { 2 - 7 } & hM & Co & hM & Co & hM & Co \\
\hline \hline IBM01 & 180 & 180 & 495 & 430 & 750 & 711 \\
\hline IBM02 & 262 & 262 & 616 & 560 & 1841 & $\mathbf{1 4 8 3}$ \\
\hline IBM03 & 953 & 950 & 1682 & 1619 & 2402 & 2219 \\
\hline IBM04 & 529 & 530 & 1689 & 1597 & 2778 & 2507 \\
\hline IBM05 & 1708 & 1697 & 3024 & 2888 & 4306 & 3874 \\
\hline IBM06 & 889 & 890 & 1484 & 1465 & 2275 & 2204 \\
\hline IBM07 & 849 & 824 & 2188 & 2036 & 3308 & 3098 \\
\hline IBM08 & 1142 & 1140 & 2363 & 2241 & 3469 & 3240 \\
\hline IBM09 & 629 & 620 & 1670 & 1606 & 2659 & 2474 \\
\hline IBM10 & 1256 & 1249 & 2283 & 2164 & 3761 & 3305 \\
\hline IBM11 & 960 & 960 & 2321 & 2196 & 3433 & 3160 \\
\hline IBM12 & 1881 & 1872 & 3730 & 3520 & 5972 & 5384 \\
\hline IBM13 & 840 & 832 & 1661 & 1671 & 2717 & 2483 \\
\hline IBM14 & 1891 & 1816 & 3278 & 3097 & 5060 & 4263 \\
\hline IBM15 & 2598 & 2619 & 5019 & 4591 & 6623 & 5960 \\
\hline IBM16 & 1755 & 1709 & 3816 & 3745 & 6475 & 5360 \\
\hline IBM17 & 2212 & 2187 & 5395 & 5194 & 8695 & 7960 \\
\hline IBM18 & 1525 & 1521 & 2881 & 2810 & 5169 & 4435 \\
\hline \hline
\end{tabular}

TABLE II

Min-Cut Two-, Four, Eight-Way Partitioning Results With up to a 10\% Deviation From EXact Partitioning. Cells Are Assigned Non-Unit (ACTUAL) AREA

\begin{tabular}{l||c|c|c|c|c|c}
\hline \multicolumn{1}{c||}{ Circuit } & \multicolumn{2}{c|}{ 2-way } & \multicolumn{2}{c|}{ 4-way } & \multicolumn{2}{c}{ 8-way } \\
\cline { 2 - 7 } & hM & Co & hM & Co & hM & Co \\
\hline \hline IBM01 & 217 & 215 & 343 & 340 & 606 & 573 \\
\hline IBM02 & 266 & 247 & 470 & 399 & 833 & 762 \\
\hline IBM03 & 707 & 608 & 1348 & 1220 & 1981 & 1879 \\
\hline IBM04 & $\mathbf{4 4 0}$ & 438 & 1321 & 1209 & 2408 & 2241 \\
\hline IBM05 & 1716 & 1681 & $\mathbf{3 0 0 2}$ & 2895 & $\mathbf{4 3 3 1}$ & 3950 \\
\hline IBM06 & 367 & 363 & 1149 & 1056 & 1716 & 1688 \\
\hline IBM07 & 716 & 721 & 1539 & 1480 & 2918 & 2707 \\
\hline IBM08 & 1149 & 1120 & 2143 & 1992 & 3330 & 3120 \\
\hline IBM09 & 523 & 519 & 1418 & 1334 & 2337 & 2079 \\
\hline IBM10 & 769 & 734 & 1845 & 1636 & 3098 & 2751 \\
\hline IBM11 & 697 & 688 & 1893 & 1699 & 2948 & 2768 \\
\hline IBM12 & 1975 & 1970 & 3577 & 3402 & $\mathbf{4 9 5 7}$ & 4762 \\
\hline IBM13 & 859 & 832 & 1698 & 1568 & 2439 & 2298 \\
\hline IBM14 & $\mathbf{1 5 2 0}$ & 1494 & 3048 & 2869 & $\mathbf{4 8 3 3}$ & 4360 \\
\hline IBM15 & $\mathbf{1 7 8 6}$ & 1771 & 4435 & 4314 & 6111 & 5756 \\
\hline IBM16 & $\mathbf{1 6 8 1}$ & 1639 & $\mathbf{3 5 6 2}$ & 3149 & 5580 & $\mathbf{5 1 4 6}$ \\
\hline IBM17 & 2252 & 2156 & 4824 & 4393 & 8222 & $\mathbf{7 0 0 3}$ \\
\hline IBM18 & $\mathbf{1 5 2 0}$ & 1520 & $\mathbf{3 1 0 4}$ & 2941 & 4833 & 4416 \\
\hline \hline
\end{tabular}

(real) cell area with CoMHP (Co) and hMETIS (hM). Out of the 108 tests executed, hMETIS outperforms or yields the same results as CoMHP in eight instances, while CoMHP outperforms hMETIS in 100 instances. For two-way partitioning, the improvements of CoMHP over hMETIS are not significant. For four-way and eight-way partitioning, CoMHP can get up to a $15 \%$ improvement in the hyperedge cuts over hMETIS. For hMETIS, Tables I and II report the best solution of bisection or hMETIS-Kway. In 102 cases, hMETIS with bisection found the best solution while hMETIS-Kway found the best solution in the six other instances.

Tables III and IV present the runtimes (parallel computational time) of both algorithms. For CoMHP, the runtime indicates the
TABLE III

Run-Time Performance For Min-Cut Two-, Four, Eight-Way PARTITIONING With UP TO A 10\% DEVIATION FROM EXACT PARTITIONING. Cells Are Assigned Unit Area

\begin{tabular}{l||c|c|c|c|c|c}
\hline \multicolumn{1}{c||}{ Circuit } & \multicolumn{2}{c|}{ 2-way } & \multicolumn{2}{c|}{ 4-way } & \multicolumn{2}{c}{ 8-way } \\
\cline { 2 - 7 } & hM & Co & hM & Co & hM & Co \\
\hline \hline IBM01 & 0.2 & 5 & 0.3 & 7 & 0.5 & 11 \\
\hline IBM02 & 0.4 & 10 & 0.7 & 12 & 1.1 & 21 \\
\hline IBM03 & 0.4 & 16 & 0.8 & 17 & 1.1 & 25 \\
\hline IBM04 & 0.5 & 16 & 1.0 & 19 & 1.3 & 26 \\
\hline IBM05 & 0.7 & 18 & 1.2 & 24 & 1.6 & 30 \\
\hline IBM06 & 0.6 & 21 & 1.2 & 23 & 1.7 & 33 \\
\hline IBM07 & 1.1 & 32 & 2.0 & 38 & 2.6 & 53 \\
\hline IBM08 & 1.6 & 36 & 2.6 & 51 & 3.4 & 59 \\
\hline IBM09 & 1.0 & 34 & 2.0 & 40 & 2.6 & 58 \\
\hline IBM10 & 2.2 & 56 & 3.5 & 65 & 5.0 & 91 \\
\hline IBM11 & 1.5 & 50 & 3.0 & 59 & 3.9 & 78 \\
\hline IBM12 & 1.9 & 62 & 4.6 & 73 & 5.1 & 115 \\
\hline IBM13 & 2.0 & 60 & 3.6 & 72 & 5.1 & 100 \\
\hline IBM14 & 5.9 & 79 & 9.1 & 141 & 13.0 & 169 \\
\hline IBM15 & 6.6 & 121 & 11.0 & 176 & 14.1 & 217 \\
\hline IBM16 & 7.6 & 142 & 13.3 & 192 & 19.0 & 238 \\
\hline IBM17 & 9.4 & 219 & 17.1 & 196 & 22.2 & 374 \\
\hline IBM18 & 7.7 & 178 & 15.1 & 192 & 20.4 & 301 \\
\hline \hline
\end{tabular}

TABLE IV

Run-Time Performance For Min-Cut Two-, Four, Eight-Way PARTITIONING With UP TO A 10\% DEVIATION FROM EXACT PARTITIONING. Cells Are Assigned Non-Unit (Actual) ArEA

\begin{tabular}{l||c|c|c|c|c|c}
\multicolumn{1}{c||}{ Circuit } & \multicolumn{2}{c|}{ 2-way } & \multicolumn{2}{c|}{ 4-way } & \multicolumn{2}{c}{ 8-way } \\
\cline { 2 - 7 } & hM & Co & hM & Co & hM & Co \\
\hline \hline IBM01 & 0.2 & 6 & 0.3 & 7 & 0.5 & 11 \\
\hline IBM02 & 0.3 & 10 & 0.7 & 13 & 1.0 & 20 \\
\hline IBM03 & 0.4 & 11 & 0.8 & 19 & 1.2 & 26 \\
\hline IBM04 & 0.5 & 16 & 0.9 & 18 & 1.3 & 26 \\
\hline IBM05 & 0.6 & 18 & 1.2 & 23 & 1.6 & 35 \\
\hline IBM06 & 0.5 & 15 & 1.2 & 22 & 1.7 & 35 \\
\hline IBM07 & 1.0 & 29 & 2.0 & 41 & 2.7 & 54 \\
\hline IBM08 & 1.2 & 25 & 2.2 & 35 & 3.1 & 57 \\
\hline IBM09 & 1.1 & 40 & 1.8 & 45 & 2.6 & 65 \\
\hline IBM10 & 1.7 & 52 & 3.4 & 64 & 4.9 & 93 \\
\hline IBM11 & 1.4 & 44 & 2.7 & 53 & 4.4 & 88 \\
\hline IBM12 & 2.0 & 58 & 3.8 & 75 & 5.1 & 113 \\
\hline IBM13 & 1.9 & 53 & 3.7 & 71 & 4.9 & 113 \\
\hline IBM14 & 6.0 & 81 & 9.0 & 145 & 13.0 & 151 \\
\hline IBM15 & 5.6 & 111 & 12.0 & 160 & 14.2 & 197 \\
\hline IBM16 & 6.7 & 168 & 13.1 & 197 & 18.0 & 264 \\
\hline IBM17 & 11.2 & 243 & 18.2 & 286 & 23.8 & 354 \\
\hline IBM18 & 8.7 & 189 & 15.9 & 235 & 20.5 & 296 \\
\hline \hline
\end{tabular}

total time to run ten iterations of $p_{0}$ plus the time to perform the coarsening phase. For hMETIS we report the time to execute one run of the bisection approach in order to factor the use of several processors by CoMHP. This biases the results slightly in favor of hMETIS given that CoMHP uses ten processors only for a few problem instances.

As can be seen from Tables III and IV, on average hMETIS is 20 to 25 times faster than CoMHP for the 108 tests. A time-optimized implementation of CoMHP can improve on the current prototype in the following ways. The outer loop of CoMHP has only a few sequential dependencies, therefore it can be easily parallelized. Though this parallelization will not reduce the work ratio between CoMHP and other partitioners, it will considerably improve the time ratio. Secondly, the 
TABLE V

COMPARING HYPEREDGE CUTS BETWEEN CoHMP AND ChMETIS (COLUMNS "Co" AND "Ch" STAND, RESPECTIVELY, FOR CoHMP AND ChMETIS). Up to a 10\% Deviation From EXAct Partitioning. Cells ArE ASSIGNED UNIT AREA

\begin{tabular}{l||c|c|c|c|c|c}
\hline \hline \multicolumn{1}{c||}{ Circuit } & \multicolumn{2}{c|}{ 2-way } & \multicolumn{2}{c|}{ 4-way } & \multicolumn{2}{c}{ 8-way } \\
\cline { 2 - 7 } & Co & Ch & Co & Ch & Co & Ch \\
\hline \hline IBM01 & 180 & 180 & 430 & 431 & 711 & 705 \\
\hline IBM02 & 262 & 262 & 560 & 537 & 1483 & 1492 \\
\hline IBM03 & 950 & 950 & 1619 & 1646 & 2219 & 2294 \\
\hline IBM04 & 530 & 527 & 1597 & 1573 & 2507 & 2534 \\
\hline IBM05 & 1697 & 1703 & 2888 & 2905 & 3874 & 3875 \\
\hline IBM06 & 890 & 892 & 1465 & 1467 & 2204 & 2222 \\
\hline IBM07 & 824 & 824 & 2036 & 2033 & 3098 & 3113 \\
\hline IBM08 & 1140 & 1140 & 2241 & 2266 & 3240 & 3217 \\
\hline
\end{tabular}

amount of improvement in the hyperedge cuts of CoMHP is not significant after two or three iterations of the search phase by process $p_{0}$. At that point the energy function (4) is low and seems stable in its minimum. Running the current prototype implementation of CoMHP only two or three iterations will not result in any serious degradations of the results obtained using ten iterations, which means we can get similar results as in Tables I and II with only about $1 / 5$ to $1 / 3$ of run times as in Tables III and IV. Thirdly, the computational time of CoMHP is dominated by the execution of the global and local search subroutines. We believe we can reduce the time spent in the global and local searches by adapting these routines to CoMHP, for example, by not flipping all vertices for refinement, but rather stopping the search after flipping part (20\%, for example) of the vertices. However, even if all these optimizations were realized, it is obvious that CoMHP will not be faster than hMETIS, or other partitioners for that matter, given that CoMHP uses repeatedly those partitioners as subroutines. On the other hand, with the same amount of computing resources as given to CoMHP (when run for ten iterations of $p_{0}$ ), hMETIS did not improve noticeably the quality of partitionings reported in Tables I and II. The situation is, however, different when hMETIS is embedded in CoMHP. Computational results from a "cooperative hMETIS" to be called ChMETIS are reported in Table V for netlists IBM01 to IBM08. The computation of these hyperedge cuts is based on the procedure of Fig. 4 after replacing the local partitioners FMS and PFM by calls to hMETIS (except for line 2a, since search after interpolation starts from an initial partitioning). The quality of hyperedge cuts produced by ChMETIS is very close to the quality of those produced by CoMHP. Since hMETIS is faster than FMS and PFM, computational times were about $10 \%$ to $20 \%$ better than CoMHP. Table V clearly demonstrates that the cooperation scheme plays a key role in the quality of the solutions produced by CoMHP. The improvement in the quality of the solutions produced by CoMHP is to a large extent independent of the partitioners used in the implementation of CoMHP.

\section{SUMMARY AND DISCUSSION}

We have explored two objectives: design an approach for the netlist partitioning problem using the cooperative multilevel search paradigm introduced by Toulouse et al. [18] and study the effectiveness of this paradigm for solving combinatorial optimization problems, in particular, those arising in the VLSI CAD area. We have presented the design and parallel implementation of an algorithm, called CoMHP, for the netlist partitioning problem. In this algorithm we combine the multilevel paradigm and the cooperative search paradigm and take advantage of the good features of both these paradigms. To date, the most successful approach to the netlist partitioning problem has been the multilevel algorithm hMETIS of Karypis et al. [11] which formulates the netlist partitioning problem as a hypergraph partitioning problem. So, we have chosen this algorithm for a comparative evaluation of the quality of solutions produced.

In CoMHP, each level is associated with a coarsened (appropriately reduced) hypergraph and a search program derived from known heuristics such as the FM heuristics. These programs execute searches on the coarsened hypergraphs at their respective levels. A distinguishing feature of CoMHP is the use of a cooperation protocol to control the coarsening of the hypergraphs at the different levels. This involves the use of three cooperation operators. The effectiveness of the algorithm depends on the specification and implementation of these operators. They control the coarsening which impacts the solution subspaces explored at the different levels. We have been conservative in exploiting this aspect of the cooperation strategy. Improvements both in terms of computational time and quality of partitionings will result from the choice of elite solutions (those selected at each level for information sharing), the choice of operators for refinement, and the selection of the levels between which cooperation takes place.

Our cooperative search paradigm can be applied to create partitioning methods capable of partitioning hypergraphs with fixed vertices, which could enhance the usefulness of this paradigm in VLSI design. The refinement phase of CoMHP is flexible and can adapt to local constraints imposed on coarsening by specific needs from the physical design process.

In the case of CoMHP, each iteration of the slowest process executes hMETIS, FM, and FMS as subroutines. It is therefore not surprising that CoMHP takes considerably longer than any of its subroutines. On the other hand, our work supports the hypothesis that individual search algorithms, with the same amount of computing resources as the cooperative computation (through restarts or other means), cannot match the performance of a successful cooperative algorithm. Based on the results presented in this paper, we believe that multilevel design provides such a successful approach to develop cooperation protocols. The cooperative multilevel search paradigm in combination with other heuristics will help produce solutions with better quality than those obtained by the original heuristics. This paradigm will also be useful to design algorithms for other combinatorial optimization problems (besides partitioning) arising in the VLSI CAD area. Our work in this paper is the first study to demonstrate this.

\section{ACKNOWLEDGMENT}

The authors wish to thank $\mathrm{C}$. Alpert and the anonymous referees for their helpful comments. 


\section{REFERENCES}

[1] C. J. Alpert, J.-H. Huang, and A. B. Kahng, "Multilevel circuit partitioning," in Proc. 34th ACM/IEEE Design Automation Conf., June 1997, pp. 530-533.

[2] C. J. Alpert and A. B. Kahng, "Recent developments in netlist partitioning: A survey," Integration: The VLSI J., vol. 19, pp. 1-81, 1995.

[3] S. H. Clearwater, B. A. Huberman, and T. Hogg, "Cooperative solution of constraint satisfaction problems," Science, vol. 254, pp. 1181-1183, 1991.

[4] J. Cong and M. L. Smith, "A parallel bottom-up clustering algorithm with applications to circuit partitioning in VLSI design," in Proc. 30th ACM/IEEE Design Automation Conf., June 1993, pp. 755-760.

[5] A. Dasdan and C. Aykanat, "Two novel circuit partitioning algorithms using relaxed locking," IEEE Trans. Computer-Aided Design, vol. 16, pp. 169-178, Feb. 1997.

[6] M. R. Garey and D. S. Johnson, Computer and Intractability: A Guide to the Theory of NP-Completeness. New York: W. H. Freeman, 1979.

[7] A. Gupta, "Fast and effective algorithms for graph partitioning and sparse matrix ordering," IBM T. J. Watson Research Center, Rep. RC 20496, 1995.

[8] T. Hogg and C. Williams, "Solving the really hard problems with cooperative search," in Proc. 11th National Conf. Artificial Intelligence (AAAI93): AAAI Press, Aug. 1993, pp. 231-236.

[9] J. J. Hopfield, "Neural networks and physical systems with emergent collective computational abilities," in Proc. National Acad. Sci. U.S., vol. 79, 1982, pp. 2554-2558

[10] B. A. Huberman, "The performance of cooperative processes," Physica $D$, vol. 42, pp. $38-47,1990$.

[11] G. Karypis, V. Aggarwal, V. Kumar, and S. Shekhar, "Multilevel hypergraph partitioning: Application in VLSI domain," IEEE Trans. VLSI Syst., vol. 7, pp. 69-79, Mar. 1999.

[12] G. Karypis and V. Kumar, "Multilevel $k$-way hypergraph partitioning," in Proc. 36th ACM/IEEE Design Automation Conf:: Association for Computing Machinery, June 1999, pp. 343-348.

[13] K.-G. Lee and S.-Y. Lee, "Efficient parallelization of simulated annealing using multiple Markov chains: An application to graph partitioning," in Proc. 1992 Int. Conf. Parallel Processing. New York: CRC, Aug. 1992, pp. III 177-III 180.

[14] D. Levine, "A parallel genetic algorithm for the set partitioning problem," in Meta-Heuristics: Theory and Applications. New York: Kluwer, 1996, pp. 23-35.

[15] L. A. Sanchis, "Multiple-way network partitioning," IEEE Trans. Comput., vol. 38, pp. 62-81, Jan. 1989.

[16] V. Schnecke and O. Vornberger, "An adaptive parallel genetic algorithm for VLSI-layout optimization," in Proc. Fourth Workshop Parallel Problem Solving From Nature. New York: Springer-Verlag, 1996, pp. 859-868.

[17] M. Toulouse, T. G. Crainic, and B. Sansó, "Self-organization in cooperative tabu search algorithms," in 1998 IEEE Int. Conf. Systems, Man, Cybernetics, Oct. 1998, pp. 2379-2385

[18] M. Toulouse, K. Thulasiram, and F. Glover, "Multi-level cooperative search," in 5th Int. Euro-Par Parallel Processing Conf., vol. 1685, Lecture notes in Computer Science. New York, Aug. 1999, pp. 533-542.

Min Ouyang received the B.S. degree from Fudan University, China, in 1990 , the M.S. degree from Peking University, China, in 1996, and the Ph.D. degree from the University of Nebraska, Lincoln, in 2000, all in in computer science.

He joined Synopsys, Inc., in 2000, where he was a Senior Research and Development Engineer in the physical synthesis group. His research interests include $\mathrm{CAD}$ of integrated circuit and systems, with particular emphasis on placement algorithms.
Michel Toulouse received the M.Sc. degree in computer science from Université de Montréal, Montréal, Canada, in 1989 and the Ph.D. degree in computer science and engineering from École Polytechnique, Université de Montréal, in 1996.

$\mathrm{He}$ is currently an Assistant Professor in the Department of Computer Science at the University of Manitoba, Canada. His research interests include metaheuristic algorithms, parallel and distributed algorithms, multilevel algorithms with applications in VLSI CAD area, and nonstandard computing paradigms (emergent computation, molecular computation, and quantum computing). $\mathrm{He}$ is co-author of a book on parallel metaheuristics.

Dr. Toulouse is an Associate Editor of the Journal of Heuristics.

Krishnaiyan Thulasiraman (F'90) received the Ph.D. degree in electrical engineering from the IIT, Madras, India, in 1968.

He holds the Hitachi Chair and is Professor in the School of Computer Science at the University of Oklahoma, Norman, where he has been since 1994. Prior to joining the University of Oklahoma, he was a Professor (1981-1994) and Chair (1993-1994) of the Electrical and Computer Engineering Department at Concordia University, Montreal, Canada. He was on the faculty in the Electrical Engineering and Computer Science Departments of the IIT during 1965 through 1981. His research interests have been in graph theory, combinatorial optimization, and algorithms and applications in a variety of areas in computer science and electrical engineering. He has published more than 100 papers in archival journals, coauthored (with M. N. S. Swamy) two text books Graphs, Networks, and Algorithms (New York: Wiley Inter-Science, 1981) and Graphs: Theory and Algorithms (New York: Wiley Inter-Science, 1992), authored two chapters in the Handbook of Circuits and Filters (New York: CRC and IEEE, 1995), and recently edited the circuit theory section of a forthcoming encyclopedia. He has held visiting positions at the Tokyo Institute of Technology, University of Karlsruhe, University of Illinois at Urbana-Champaign and Chuo University, Tokyo.

Dr. Thulasiraman has received several awards and honors: IEEE CAS Society Golden Jubilee Medal (1999), Senior Research Fellowship of the Japan Society for Promotion of Science (1988), and Guest Professorship of the German National Science Foundation (1990). He has been Vice President (Administration) of the IEEE CAS Society $(1998,1999)$, Technical Program Chair of ISCAS (1993, 1999), Co-Guest Editor of a special issue on "Computational Graph Theory: Algorithms and Applications" in IEEE TRANSACTIONS ON CIRCUITS AND SYSTEMS, (March 1988), Associate Editor of the IEEE TRANSACTIONS ON CIRCUITS AND SYSTEMS (1989-1991, 1999-2001), and Founding Regional Editor of the Journal of Circuits, Systems, and Computers. Recently, he founded the Technical Committee on Graph Theory and Computing of the IEEE CAS Society.

Fred Glover is the MediaOne Chaired Professor in Systems Science at the University of Colorado, Boulder, and Distinguished Researcher and co-founder of the Hearin Center for Enterprise Science at the University of Mississippi. He has authored or coauthored more than 300 published articles and five books in the fields of mathematical optimization, computer science, and artificial intelligence. He serves on the advisory boards of several organizations is a co-founder of Heuristic, Inc. and OptTek Systems, Inc.

Dr. Glover is the recipient of the distinguished von Neumann Theory Prize, as well as of numerous other awards and honorary fellowships, including those from AAAS, NATO, INFORMS, DSI, CAS, DCA, ERI, AACSB, and a variety of others. He is a member of the National Academy of Engineering.

Jitender S. Deogun received the B.S. (Hons.) degree from Punjab University, Chandigarh, India, in 1967, and the M.S. and Ph.D. degrees from University of Illinois, Urbana, in 1974 and 1979, respectively.

Since 1981, he has been a Professor of Computer Science and Engineering at the University of Nebraska, Lincoln. His current research interests include structural and algorithmic graph theory, combinatorics, design and analysis of algorithms, optical networks, and information retrieval. 